\title{
Effect of Antacid Concentration on Acetaminophen-Alginate Sphere Diffusion in a Model Gastric System
}

\author{
Mustafaa Munir ${ }^{1}$, Ben Beasley ${ }^{1}$ and Alexander Smith ${ }^{1}$ \\ ${ }^{1}$ Fishers High School, Fishers, IN, USA
}

\section{$\underline{\text { ABSTRACT }}$}

This paper presents a growing awareness for socio-cultural and socio-economic factors in medical and pharmaceutical treatments through a review of the current literature, and a concurrent need to produce a cheap and vegetarian alternative to current capsules. It was hypothesized that increasing the concentration of antacid would increase the rate of diffusion of the contents of an acetaminophen-alginate sphere due to the neutralization of $\mathrm{pH}$ between stomach acid and the antacid. The methodology employed prepared these spheres with reverse spherification, tested them via spectrophotometry in a model stomach system, and analyzed the data using the Beer-Lambert Law. Finally, it was found that increasing antacid concentration in the form of calcium carbonate has a direct, yet exponentially decreasing relationship with the rate of diffusion, therefore the hypothesis was only partially correct.

\section{Introduction}

Within the realm of medication, there exists more than solely a medical outlook in treating a given population or patient - treatment is inherently a holistic process. It is because of this humanistic perspective that it has been increasingly important to consider medication through both a socio-cultural and socio-economic lens in conjunction with a pure scientific lens. For example, the World Health Organization (WHO) in their Guidelines on Country Pharmaceutical Pricing Policies has stated that medication pricing has become increasingly problematic in ensuring treatment (WHO, n.d.). Additionally, researchers writing for Research in Social and Administrative Pharmacy (RSAP) have found that socio-cultural perspectives directly affect medicine use and commitment (Daher et al., 2015). The purpose of the following literature review will be to discuss the socio-cultural and socio-economic needs for developing a method of acetaminophen-antacid delivery with the use of alginate, as well as scientific backing to establish hypothesis-based research and methods.

\section{Literature Review}

\section{Socio-Cultural and Socio-Economic Need for Development}

The socio-economic pitfalls to medication do not only apply to newer, recently researched drugs, but also for common, everyday drugs like acetaminophen or antacids. A research paper in Frontiers in Medicine, citing the WHO, found that access to essential medicines such as the two previously mentioned is problematic for a full third of the global population. This problem has also been recently increasing in middle-income countries in addition to lesser-developed nations (Stevens \& Huys, 2017). Many drugs, including acetaminophen, come in the form of a capsule that dissolves in the stomach and releases its contents to be absorbed into the bloodstream. It is here where the socio-economic need for an alginic form of drug delivery interacts with the socio-cultural need due to the presence of gelatin in capsules 
(Merck, n.d.). Additionally, 50-80\% of capsules contain animal gelatin, which is commonly derived from either a porcine (pig) or bovine (cow) source. Due to dietary restrictions posed by many of the world's religions, there exists widespread objections to the use of animal-derived materials in medication. In a BMC Medical Ethics study, it was found that from the ten religious branches that responded to their inquiry, Sunni Muslims, Shi'a Muslims, Vaishnava Hindus, and Sikhs have an objection to porcine-derived and/or bovine-derived materials in medication (Eriksson $e t$ al., 2013). One specific example of the interaction of the socio-economic and socio-cultural needs is the Halal food market, the market based on the Islamic dietary tradition. In 2013, the Halal food market was a 580 to 660 -billiondollar industry with gelatin being one of the most studied ingredients for use and source authentication (Hameed et al., 2018). However, current alternatives to gelatin in drug delivery such as vegetarian-sourced capsules can cost five times as much due to the use of synthetic compounds such as Hypromellose, a synthetic cellulose (Jain et al., 2012).

Although acetaminophen is commercially available in tablet form and does not have gelatin in such a form, there is a different issue with tablets beyond dietary restrictions that should be considered. The tablet manufacturing process is expensive, with tablet pressing requiring an expensive cost of entry with a tablet pressing apparatus. This barrier exists even if dry compression, the simplest form of tablet manufacturing, is used over more complex methods such as wet or dry granulation (Eyjolfsson, 2015). The cost barrier that tablet pressing presents brings up the socioeconomic concern of developing areas who may wish to pursue domestic or local manufacturing of drugs instead of relying on outside sources out of desire or necessity.

\section{Scientific Background and Current Research}

Alginate is a natural compound derived from brown seaweed and has been found to be highly biocompatible with humans, have a relatively low cost, and have applications in fields ranging from molecular gastronomy to biomedicine. Many of alginate's applications come from its gelation when in contact with divalent cations such as calcium, allowing it to cross-link and form polymers in what is commonly called spherification. Spherification allows for a solution to be encapsulated in an alginate polymer sphere, commonly used in foods and drinks (Lee \& Mooney, 2012). There are three main types of spherification: basic, where alginate is added to the solution with a divalent cation bath; reverse where divalent cations are added to the solution with an alginate bath; and frozen reverse, which is the former with a frozen solution and heated bath (Potter, 2010). However, alginic spheres have been used in the field of medicine as well, with acetaminophen-alginate spheres successfully being formed in a study at the University of Puerto Rico (Rubio \& Ghaly, 1993). Additionally, in a study that involved the encapsulation of proteins in alginate spheres, it was found that a more acidic $\mathrm{pH}$ was correlated with a higher release of the contents of an alginate sphere compared to a less acidic and neutral $\mathrm{pH}$ (Zhang et al., 2016).

Acetaminophen is one of the most used drugs for pain treatment and fever reduction, with the most common form being taken in orally. When taken orally, acetaminophen tends to be dissolved and taken into the bloodstream faster in the less acidic environment of the intestines compared to the highly acidic stomach, however, most acetaminophen still is taken in at the stomach (NCBI, 2021). Acetaminophen is also readily dissolvable in water, allowing for experimentation to take advantage of water as a universal medium (Granberg \& Rasmuson, 1999). As acetaminophen has documented spectroscopically based data, the concentration of acetaminophen can also be determined with the use of the Beer-Lambert Law, which states that concentration is directly proportional with absorbance (Clark \& Gunawardena, 2020).

Antacids are the medical applications of $\mathrm{pH}$ buffers, where they neutralize the stomach's secreted acid to alleviate conditions such as heartburn. Like most other drugs, a tablet antacid would have less of an effect compared to a liquid antacid due to the immense decrease in surface area in the case of a tablet relative to a liquid. When combined with alginates, some antacids can form a compound that protects the esophagus from stomach acid secretions in conditions such as acid reflux (International Foundation for Gastrointestinal Disorders [IFFGD], 2019). In research concerning antacids, it has been found that the neutralization of stomach acids can increase the absorption of 
certain drugs. This would have clinical significance in speeding up the onset of pain relief in the case of pain treating medications, such as acetaminophen (Neuvonen \& Kivistö, 1994).

\section{Literature Review Conclusion}

The holistic perspective in treating the world's people lies in medication, as well as an improvement and/or concern for the socio-economic and socio-cultural factors that interact with medication. This literature review had the goal of demonstrating the need to develop a sustainable, vegetarian, and low-cost method of administering commonly taken drugs such as acetaminophen or antacids in order to treat pain, fever, and anything arising from high amounts of stomach acid. The reviewed literature has shown that there exists a potential solution in the use of alginate spheres in the administration of these drugs to those affected by the previously detailed socio-economic and socio-cultural factors. In furthering this literature review, experimentation with alginate, antacids, and acetaminophen will explore the viability of this potential solution with the research question - to what extent does the concentration of antacid in an acetaminophen-alginate sphere have an effect on the rate of diffusion of the sphere's contents?

\section{Methods}

\section{Experimental Overview}

Given the nature of the research question, the methodology employed in this investigation was experimentally based around the scientific method. Therefore, the independent variable was differing amounts of antacid added to an acetaminophen-alginate sphere. The dependent variable was the rate of diffusion of the spherical contents, and some of the controlled variables included the sodium alginate bath, amount of acetaminophen used, amount of calcium chloride used, concentration of hydrochloric acid, and equipment used.

Three hypotheses were formulated, one "real" hypothesis, an alternative hypothesis, and a null hypothesis. The latter two were used for probability value ( $p$-value) testing in the discussion.

\section{Hypothesis}

If the concentration of antacids were to increase in an acetaminophen-alginate sphere, then the rate of diffusion of the contents will be increased because antacids are basic compounds that neutralize acidic compounds and have been shown to increase drug absorption in the stomach when taken (Neuvonen \& Kivistö, 1994).

\section{Alternative Hypothesis}

There exists a correlation between antacid concentration and the rate of diffusion of spherical contents.

\section{Null Hypothesis}

There does not exist a correlation between antacid concentration and the rate of diffusion of spherical contents.

\section{Materials Used}

Sodium alginate (TICA-algin® 400, TIC GUMS-brand, manufacturing country unknown), calcium chloride (Willpowder-brand, manufacturing country unknown), acetaminophen (liquid, cherry flavored, red, CVS-brand, manufacturing country unknown), antacid tablets (mint flavored, calcium carbonate, Kroger-brand, manufacturing country unknown), hydrochloric acid (1 M), water, UNICO 1000 spectrophotometer, cuvettes, Corning hot plate, blender, 
thermometer, freezer, refrigerator, Scout Pro digital mass scale, mortar and pestle, beakers $(50 \mathrm{~mL}, 600 \mathrm{~mL})$, graduated cylinders $(25 \mathrm{~mL})$, weigh boats, spot plates $(1 \mathrm{~g})$, pipette pump, disposable pipette $(2 \mathrm{~mL})$, scoopula, stirring rod, sieve, plastic wrap, tape, and parafilm were collected for the execution of the methodology.

\section{Solution Preparation}

First, a $0.5 \%$ solution of sodium alginate was prepared with $500 \mathrm{~mL}$ of water and $2.50 \mathrm{~g}$ of sodium alginate. Both were mixed in a blender until the sodium alginate was fully dissolved. This solution was then transferred to a $600 \mathrm{~mL}$ beaker, covered with labelled parafilm, and placed in a refrigerator overnight to eliminate air bubbles.

Next, five solutions were prepared which were the five tested levels of the independent variable. First, $15 \mathrm{~mL}$ of liquid acetaminophen and $15 \mathrm{~mL}$ of water were measured and mixed in a $50 \mathrm{~mL}$ beaker to dilute the acetaminophen and allow later solutes to be dissolved easier. Second, $0.30 \mathrm{~g}$ of calcium chloride was mixed into the diluted acetaminophen solution to achieve a $1 \%$ concentration of calcium chloride. Finally, differing amounts of antacids were mixed into the solution depending on which solution was being created (control, $200 \mathrm{mg}, 400 \mathrm{mg}, 600 \mathrm{mg}$, or $800 \mathrm{mg}$ of antacid, corresponding to $0 \mathrm{M}, 0.07 \mathrm{M}, 0.13 \mathrm{M}, 0.20 \mathrm{M}$, and $0.27 \mathrm{M}$ of antacid concentration respectively). Antacid was not added to the control solution. Antacid tablets were crushed into a fine powder, measured to the desired mass, and added in the appropriate amount to the dilute acetaminophen solution with $1 \%$ calcium chloride. All previous steps in this paragraph were repeated four more times to create all five solutions listed above.

Next, to complete the solution preparation, $2 \mathrm{~mL}$ of each solution was measured into a spot plate. This was done a total of six times for each solution across two spot plates for a total of 30 spots. They were also labelled appropriately with each solution (control, $200 \mathrm{mg}$, etc.). Finally, the filled spot plates were covered in plastic wrap and placed in the freezer on the coldest possible setting to freeze the solutions for later testing.

\section{Diffusion Testing}

The second stage of the methodology was to test the diffusion of the capsules' contents using spectrophotometry. Of the six spots for each solution, four were used in the reverse spherification method, one was used in the frozen reverse spherification method, and one was used as a potential extra if blatant error was observed. First, the hydrochloric acid was diluted from $1 \mathrm{M}$ down to $0.01 \mathrm{M}$ by mixing $3 \mathrm{~mL}$ of $1 \mathrm{M}$ hydrochloric acid and $300 \mathrm{~mL}$ of water. This was done to dilute the hydrochloric acid down to a $\mathrm{pH}$ of 2.0 which served as a rough model system of the stomach based on the average $\mathrm{pH}$ of gastric acid (Marieb \& Hoehn, 2019). Next, the $0.5 \%$ sodium alginate solution was brought out of the refrigerator and placed on a hot plate set on medium without the parafilm. The solution was left to heat up to about 50 degrees Celsius according to a thermometer.

While this occurred, the spectrophotometer was turned on and set up according to manufacturer recommendations (UNICO, n.d.). The spectrophotometer was zeroed around the wavelength for visible red light (around 700 $\mathrm{nm}$ ) as the prepared solutions were all red. The spectrophotometer was set on transmittance as opposed to absorbance, which was done to make zeroing easier. Zeroing was accomplished with pure water, a cuvette, and necessary adjustments to the transmittance $0 \%$ knob.

Next, the frozen solutions were taken out of the freezer and uncovered. For the first five trials, the spot plates were placed immediately back into the freezer and rotated between the two to allow for frozen reverse spherification testing. For the later 20, both plates were left out until the capsules had thawed for simple reverse spherification testing. A $1 \mathrm{~g}$ scoop was used to submerge one spot of solution into the sodium alginate solution for about 30 seconds until thawed and spherified. The capsule was then transferred into a beaker for degradation. $10 \mathrm{~mL}$ of the dilute hydrochloric acid was poured into the beaker to submerge the capsule. After 10 seconds, the beaker's contents without any alginic residue were poured into a cuvette and the transmittance reading was recorded. All steps in this paragraph were repeated 24 times for each spot on the spot plate, with the next four being done with the frozen solutions, while the last 
20 were done with the room temperature solutions. There were no edits in procedure between frozen and room temperature solutions as the $1 \mathrm{~g}$ scoop was able to gather all solution in the spot plate with ease.

\section{Results}

Data analysis heavily revolved around the usage of the Beer-Lambert Law and an understanding of its variables. The law itself commonly is used in the form of an equation (1).

Equation 1: Beer-Lambert Law:

$$
A=\varepsilon \ell c
$$

In the equation, $A$ represents absorbance, $\varepsilon$ represents the molar extinction coefficient, $\boldsymbol{\ell}$ represents the optical path length, and $c$ represents the concentration. Due to the experimental design of testing the color of the same solution (acetaminophen-alginate spherical contents) in the same spectrophotometer for all trials, both the molar extinction coefficient which is specific to a given solution and the optical path length were kept constant as controlled variables. Therefore, when deriving the equation, if the molar extinction coefficient and optical path length are kept constant, it can be said that the change of absorbance over time is directly related to the change of concentration over time (2) (Clark \& Gunawardena, 2020).

Equation 2: Derivation of Beer-Lambert Law:

$$
\frac{d A}{d t}=\frac{d c}{d t}
$$

The raw data from this investigation was entirely found in percent transmittance, with statistical processing of that data detailed in Table 1. The frozen reverse spherification trial resulted in outlier data and was not included in Table 1 nor Figure 1.

Table 1. Transmittance $(\% T)$ of acetaminophen-alginate spherical contents in $\mathrm{HCl}(0.01 \mathrm{M})$ with differing amounts of antacid $(\mathrm{mg})$ added to the sphere. *

\begin{tabular}{lccccc}
\hline & & & \multicolumn{3}{c}{$95 \% \mathrm{CI}$} \\
$\mathrm{mg}$ & $\mathrm{x}^{-}$ & $\sigma$ & $\sigma_{\mathrm{x}^{-}}$ & $\mathrm{LCL}$ & $\mathrm{UCL}$ \\
\hline 0 & 97.50 & 0.58 & 0.15 & 96.58 & 98.42 \\
200 & 94.50 & 0.58 & 0.15 & 93.58 & 95.42 \\
400 & 89.50 & 1.00 & 0.25 & 87.91 & 91.09 \\
600 & 89.75 & 0.50 & 0.15 & 88.95 & 90.55 \\
800 & 89.25 & 1.71 & 0.43 & 86.53 & 91.97 \\
\hline
\end{tabular}

$* n=4$ for each amount of antacid, $\mathrm{x}^{-}=$sample mean $(\% T), \sigma=$ standard deviation, and $\sigma_{\mathrm{x}^{-}}=$standard error of the mean. LCL and UCL are the lower confidence and upper confidence limits for a 95\% confidence interval about the mean. Percent transmittance $(T)$ can be related to absorbance $(A)$ with a logarithmic relationship (3).

Equation 3: Logarithmic relationship between $(T)$ and $(A)$ :

$$
A=-\log T
$$


After converting percent transmittance to absorbance, it was plotted on the $y$-axis with antacid concentration on the $x$ axis in Figure 1. The data and trendline seem to show asymptotic behavior for the resultant absorbance when antacid concentration increased.

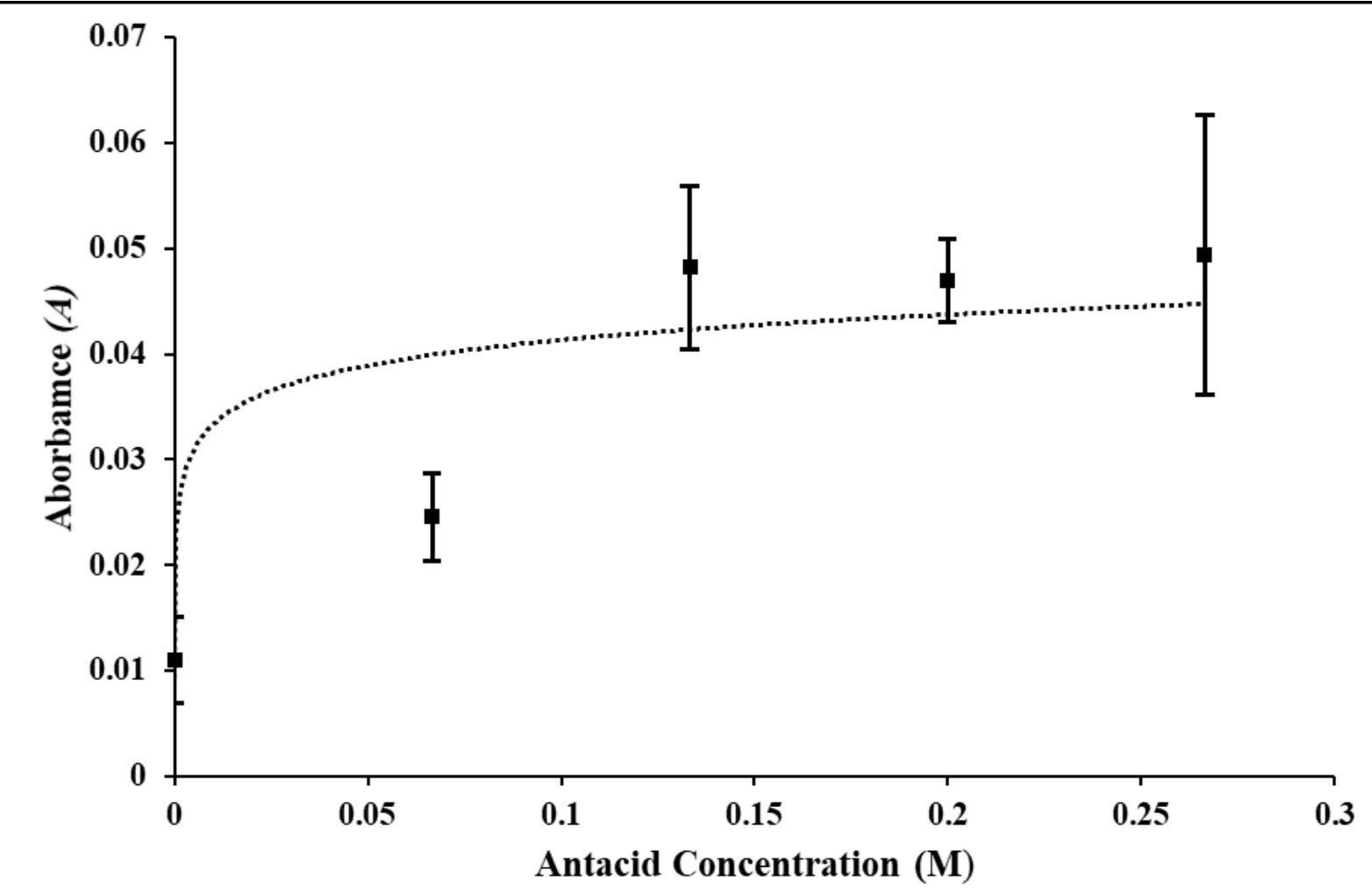

Figure 1. Absorbance $(A)$ of spherical contents in $\mathrm{HCl}(0.01 \mathrm{M})$ as a function of antacid concentration $(\mathrm{M}) . n=4$ for each level of antacid concentration and $\mathrm{R}^{2}=0.95$. The squares represent mean absorbance values and the error bars represent the LCL and UCL for a 95\% confidence interval. Note that the error bars for the first point are partially obscured by the $y$-axis.

\section{Discussion}

One-way analysis of variance (ANOVA) testing of the raw data resulted in a $p$-value below 0.00001 without the outlier data, and a $p$-value of 0.064 with the outlier data. With a standard of significance of $p<0.05$, the non-outlier data set is therefore significant, the null hypothesis can be confidentially rejected, and the alternative hypothesis is accepted for the reverse spherification trials. Applying the same standard of significance to the same set of data containing the outlier/frozen reverse spherification trial, the null hypothesis cannot be rejected. This means that there is only a significant effect of antacid concentration on the diffusion of acetaminophen-alginate spherical contents with spheres prepared with the reverse spherification method only in the scope of the experimental design used.

Using the relationship from Figure 1 in addition to the Beer-Lambert Law derivation (2) the original research question, to what extent does the concentration of antacid in an acetaminophen-alginate sphere have an effect on the rate of diffusion of the sphere's contents, can be answered. By equating the absorbance of each solution to its change of absorbance (since starting absorbance was 0 for every solution from a starting transmittance of $100 \%$, final 
absorbance would therefore equal change of absorbance), and then change of absorbance to change in concentration, it is shown that the concentration of antacid has a direct, yet exponentially decreasing relationship with the rate of diffusion of acetaminophen-alginate spherical contents. This is because the change in concentration of the tested solution would be analogous to the rate of diffusion of the contents into said solution. Additionally, this partially supports the hypothesis, which correctly predicted the direct relationship between the concentration of antacid and the rate of diffusion of acetaminophen-alginate spherical contents but not the exponentially decreasing part to it.

A possible explanation of why the direct relationship between antacid concentration and the rate of diffusion experienced an exponential decrease could be related to $\mathrm{pH}$. The $\mathrm{pH}$ of the first trial was at 2.0 since it was pure 0.01 $\mathrm{M}$ hydrochloric acid, with the next four being at 6.35, 6.65, 6.83, and 6.95, respectively. Titration curves for a strong acid and weak base (which in this case, hydrochloric acid is a strong acid and calcium carbonate is a weak base) have an equivalence point at around a $\mathrm{pH}$ of 5. This means that beyond this $\mathrm{pH}$ in either direction, the effect of weak base added has on $\mathrm{pH}$ is lessened (Chang \& Cox, 2019). Looking at the pH's of all the trials graphically, there is a stark resemblance to part of a titration curve (Figure 2).

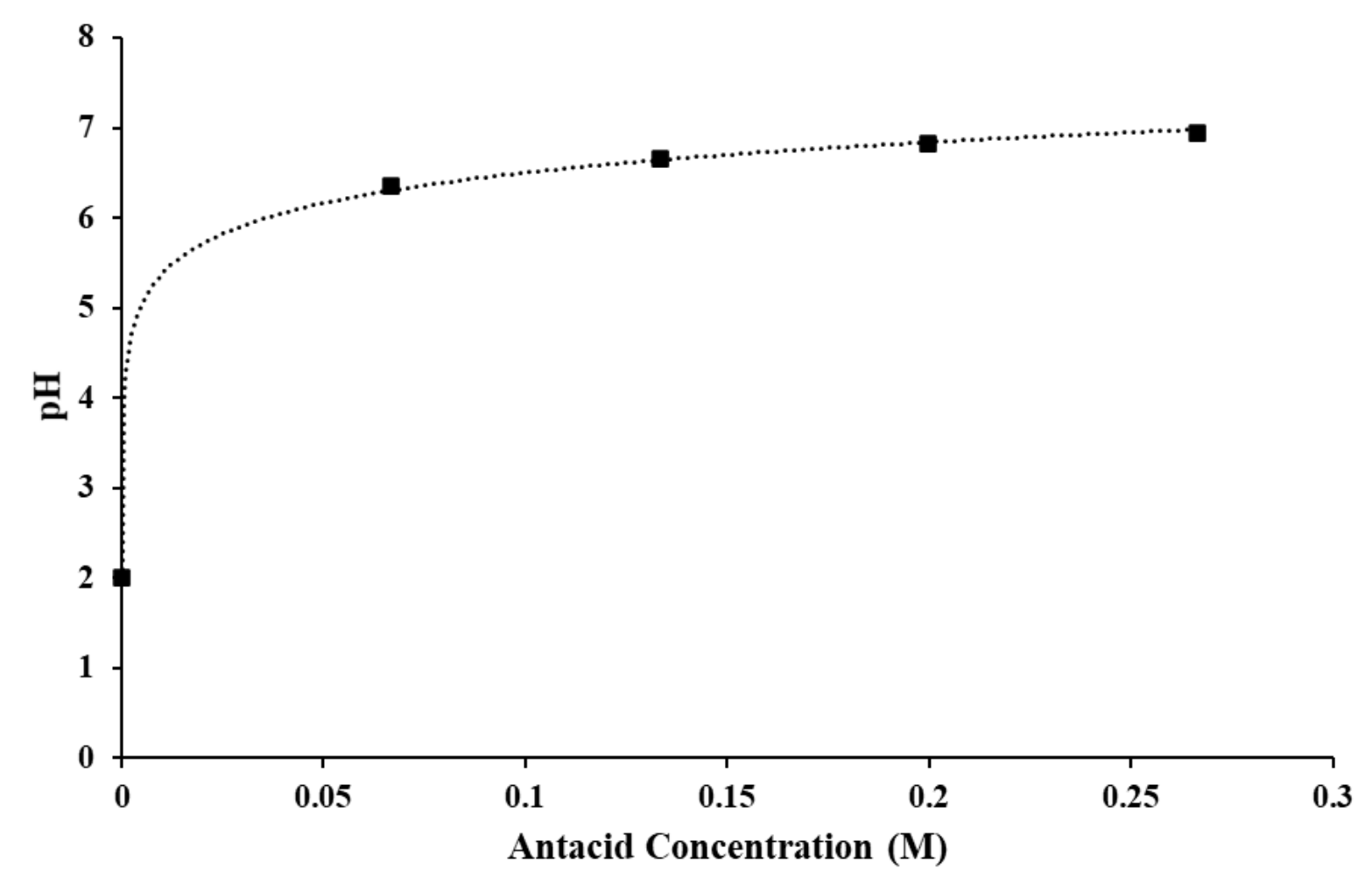

Figure 2. $\mathrm{pH}$ of solutions after the diffusion of acetaminophen-alginate spherical contents as a function of antacid concentration $(\mathrm{M}) . n=4$ for each level of antacid concentration and $\mathrm{R}^{2}=0.99$.

As seen in Figure 2, the $\mathrm{pH}$ of the trials approaches a neutral $\mathrm{pH}$ of 7 as antacid concentration is increased at a constant rate, corresponding to Figure 1 in which the absorbance (and rate of diffusion) also asymptotically approaches a value. Therefore, it can logically be claimed that the rate of diffusion of acetaminophen-alginate spherical contents approaches a practical maximum value at around a neutral $\mathrm{pH}$. This would line up with previous research that has found a correlation with antacids neutralizing stomach $\mathrm{pH}$ and a faster onset of pain relief via a quicker rate of diffusion (Neuvonen \& Kivistö, 1994). However, a limitation to this claim is that this investigation did not test 
antacid concentrations high enough to result in an overall basic $\mathrm{pH}$ above 7 and the resultant rate of diffusion of spherical contents in such an environment.

Other limitations to this investigation include both random errors from human error and systematic error from experimental design. One of the biggest glaring systematic errors alluded to throughout this paper was the inclusion of frozen reverse spherification as one of the five trials alongside reverse spherification. The frozen reverse spherification trials both qualitatively had no color change and quantitatively had no change in transmittance in the 10 second time allocated in the methods, therefore, there was no measured diffusion in any of these trials. The inclusion of this data greatly threw off calculations and significance (as shown through the immense $p$-value differences just from the inclusion) and it had to be thrown out as an outlier. This decreased the intended sample size from five to four, increasing the risk for insignificant data. Additional systematic error resulted from the usage of store-bought acetaminophen and antacid because the difficulty in acquiring pure versions of either of these compounds. The additives in either very well could have affected the rate of diffusion in a multitude of ways, and because of this limitation, this investigation can only confidently report on a correlation without specific numbers. However, the usage of the red, cherry-flavored acetaminophen made it possible for finding the rate of diffusion via the Beer-Lambert Law due to the clear presence of coloration. Also, the usage of an antacid that contained calcium (calcium carbonate) very well could have increased gelation between trials since dissolving the antacid powder would have introduced extra divalent ions. Increased gelation at higher concentrations of antacid most likely would have decreased the rate of diffusion from an increased resistance to degradation, but this was not seen in the data. Finally, another systematic error comes from the approximation of the stomach environment with pure hydrochloric acid at an average $\mathrm{pH}$. Although gastric acid is mainly comprised of hydrochloric acid, there are also potassium chloride and sodium chloride salts present. Additionally, the stomach environment contains numerous digestive enzymes, mucus linings, etc. that were not simulated through a simple $2.0 \mathrm{pH}$ solution of hydrochloric acid. Therefore, the approximation of the stomach environment also contributes to the limitation of a correlation-only conclusion detailed above.

The applications of this research must be done in cooperation with existing research because this investigation focused in on a single specific aspect to drug delivery, $\mathrm{pH}$. Existing research on acetaminophen-alginate spheres is sparse due to it being a specific drug administered via a specific, untraditional way. However, there still have been specific variables tested on the release profile of acetaminophen-alginate spheres. One paper tested polymer level, concentration of cross-linking agent (alginate), and the time the spheres spent in the cross-linking solution (alginate bath) (Rubio \& Ghaly, 1993). Another tested preparation needles, a pH polymer excipient, and a water entry excipient (Hamed et al., 2017). Although these investigations were much more advanced in scope compared to this one, a common theme between most of the tested variables in the two papers and the $\mathrm{pH}$ tested in this investigation is that there is a "maximum" value that the rate of diffusion approaches, where adding more antacid, cross-linking agents, excipients, etc. starts to have a less significant effect.

In reference to the socio-cultural and socio-economic themes presented in the literature review, an application of this specific investigation would be to move closer to the production of a valid option for a cheap and vegetarian alternative to current animal gelatin-based capsules. Additionally, while more and more research is done in the realm of alginates and drug diffusion, this research can be used in conjunction with existing research for crude estimations of specific drug release types when they are not available. Although previously and throughout this paper, the frozen reverse spherification data was deemed an outlier, it was qualitatively observed that there started to be a coloration change (therefore signaling that the acetaminophen-alginate sphere had started to diffuse) 20 minutes after submerged in the acid compared to the 10 seconds in the reverse spherification trials. Additional research in the application of frozen reverse spherification in drug diffusion very well could serve as a starting point for the development of cheap and vegetarian alternatives to extended-release and/or controlled-release capsules, both of which have a slower rate of diffusion compared to an immediate-release alternative. Also, additional research as to why the correlation found was opposite to current research regarding protein diffusion from antacid spheres would be extremely valuable in fully understanding pH's effect on alginic drug diffusion (Zhang, et al., 2016). 


\section{Conclusion}

While this investigation only confidently resulted in a simple correlational statement between two variables, it is a correlation that nonetheless contributes to the growing field of socio-cultural and socio-economic awareness in medicine and pharmaceuticals. As a result, further research must be done to correctly quantify the research done in this investigation due to its systematic errors to allow this research to become practical in pushing this field forward.

\section{Acknowledgments}

I would like to extend a huge thanks to two key individuals from Fishers High School in this paper's making. First, I would like to thank Mr. Ben Beasley for his guidance and teaching on research, and second, to Mr. Alex Smith for his subject-specific instruction and lab resources for this paper.

\section{References}

Clark, J., \& Gunawardena, G. (2019). The Beer-Lambert Law. Retrieved from Chemistry LibreTexts website: https://chem.libretexts.org/Bookshelves/Physical and Theoretical Chemistry Textbook Maps/Supplemental Modules (Physical and Theoretical Chemistry)/Spectroscopy/Electronic Spectroscopy/Electronic Spectroscopy Basics/The Beer-Lambert Law

Corning. (1995). Instruction Manual - 120V/100V [PDF File]. Retrieved February 26, 2020 from https://www.americaninstrument.com/pdf/2810-HEAT-STIR.pdf

Daher, M., Chaar, B., \& Saini, B. (2015). Impact of Patients' Religious and Spiritual Beliefs in Pharmacy: From the Perspective of the Pharmacist. Research in Social and Administrative Pharmacy, 11(1), e31-e41. https://doi.org/10.1016/j.sapharm.2014.05.004

Drug Absorption-Drugs. (n.d.). Retrieved December 19, 2019, from Merck Manuals Consumer Version website: https://www.merckmanuals.com/home/drugs/administration-and-kinetics-of-drugs/drug-absorption

Eriksson, A., Burcharth, J., \& Rosenberg, J. (2013). Animal Derived Products May Conflict with Religious Patients' Beliefs. BMC Medical Ethics, 14, 48. https://doi.org/10.1186/1472-6939-14-48

Eyjolfsson, R. (2015). Chapter Two - Conventional-Release (CR) Tablets. In Design and Manufacture of Pharmaceutical Tablets (pp. 29-41). https://doi.org/10.1016/B978-0-12-802182-8.00002-7

Granberg, R. A., \& Rasmuson, Å. C. (1999). Solubility of Paracetamol in Pure Solvents. Journal of Chemical \& Engineering Data, 44(6), 1391-1395. https://doi.org/10.1021/je990124v

Hamed, S., Ayob, F. A., Alfatama, M., \& Doolaanea, A. A. (2017). Enhancement of The Immediate Release of Paracetamol From Alginate Beads. International Journal of Applied Pharmaceutics, 9(2), 47. doi:

10.22159/ijap.2017v9i2.15672

Hameed, A. M., Asiyanbi-H, T., Idris, M., Fadzillah, N., \& Mirghani, M. E. S. (2018). A Review of Gelatin Source Authentication Methods. Tropical Life Sciences Research, 29(2), 213-227.

https://doi.org/10.21315/tlsr2018.29.2.15 
International Foundation for Gastrointestinal Disorders. (2019). Antacids - IFFGD. Retrieved December 19, 2019, from Iffgd.org website: https://www.iffgd.org/diet-treatments/antacids.html

Jain, B. V., Pawar, S. R., Patil, N. R., Patil, P. J., \& Sharma, S. N. (2012). A Vegetarian Capsule: A Review. Int. J. Pharm. Res. Technol, 2, 10-12.

Lee, K. Y., \& Mooney, D. J. (2012). Alginate: Properties and Biomedical Applications. Progress in Polymer Science, 37(1), 106-126. https://doi.org/10.1016/j.progpolymsci.2011.06.003

Chang, C., \& Cox, K. (2019). Titration of a Weak Base with a Strong Acid. Retrieved from Chemistry LibreTexts website: https://chem.libretexts.org/Ancillary Materials/Demos Techniques and Experiments/General Lab Techniques/Titration/Titration of a Weak Base with a Strong Acid

Marieb, E. \& Hoehn, K. (2019). Human Anatomy \& Physiology. Hoboken, New Jersey: Pearson Education, Inc.

National Center for Biotechnology Information (2021). PubChem Annotation Record for ACETAMINOPHEN, Source: Hazardous Substances Data Bank (HSDB). https://pubchem.ncbi.nlm.nih.gov/source/hsdb/3001

Neuvonen, P. J., \& Kivistö, K. T. (1994). Enhancement of Drug Absorption by Antacids: An Unrecognised Drug Interaction. Clinical Pharmacokinetics, 27(2), 120-128. https://doi.org/10.2165/00003088-199427020-00004

OHAUS. (n.d). Scout Pro Balance Instruction Manual [PDF File]. Retrieved February 26, 2020 from https://www.testequipmentdepot.com/ohaus/pdfs/scout-pro-manual.pdf

Potter, J. (2010). Cooking for Geeks: Real Science, Great Hacks, and Good Food. Sebastopol, California: O'Reilly.

Rubio, M. R., \& Ghaly, E. S. (1993). Acetaminophen-alginate Spheres Prepared by Cross Linking Technology. Puerto Rico Health Sciences Journal, 12(4), 269-272.

Shapley, P. (2010). Equilibria with Calcium Carbonate. Retrieved from http://butane.chem.uiuc.edu/pshapley/environmental/125/3.html

Social Science Statistics. (n.d.). One-Way ANOVA Calculator. Retrieved February 27, 2020, from https://www.socscistatistics.com/tests/anova/default2.aspx

Social Science Statistics. (n.d.). Single-Sample Confidence Interval Calculator. Retrieved February 27, 2020, from https://www.socscistatistics.com/confidenceinterval/default2.aspx

Social Science Statistics. (n.d.). Standard Deviation and Variance Calculator. Retrieved February 27, 2020, from https://www.socscistatistics.com/descriptive/variance/default.aspx

Stevens, H., \& Huys, I. (2017). Innovative Approaches to Increase Access to Medicines in Developing Countries. Frontiers in Medicine, 4, 218. https://doi.org/10.3389/fmed.2017.00218

UNICO. (n.d.). Basic Visible Spectrophotometer. Retrieved February 26, 2020, from https://www.unicosci.com/s$\underline{1000 . h t m l}$ 
WHO | Medicines Pricing and Financing. (n.d.). Retrieved December 19, 2019, from WHO website: http://www.who.int/medicines/areas/access/en/

Zhang, Z., Zhang, R., Zou, L., \& McClements, D. J. (2016). Protein Encapsulation in Alginate Hydrogel Beads: Effect of $\mathrm{pH}$ on Microgel Stability, Protein Retention and Protein Release. Food Hydrocolloids, 58, 308-315. https://doi.org/10.1016/j.foodhyd.2016.03.015 